\title{
Die Herausforderung ist eine gute Vernetzung
}

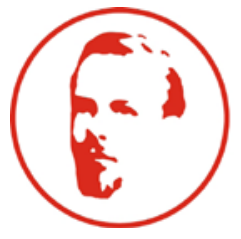

\section{DZD}

Deutsches Zentrum

für Diabetesforschung

\begin{abstract}
Gemeinsam kommt man weiter - diese Weisheit wird auch dank moderner Kommunikationsmittel in der Forschung mehr und mehr umgesetzt. Prof. Michael Roden und Prof. Martin Hrabě de Angelis vom Vorstand des Deutschen Zentrums für Diabetesforschung sehen das auch bei dieser aktuellen Studie bestätigt.
\end{abstract}

Welches Ergebnis hat Sie bei dieser Forschungsarbeit von Hernández EÁ et al. am meisten verblüfft?

Roden: Überraschend war, dass eine einzige Palmöldosis bei gesunden Menschen so schnell und direkt Auswirkungen auf die Leber hat und durch die verabreichte Fettmenge bereits eine Insulinresistenz ausgelöst wird.

Können Sie skizzieren, was neu an Ihrer wissenschaftlichen Arbeit ist? Roden: Eine Besonderheit unserer Untersuchung ist, dass wir den Leberstoffwechsel von Menschen mit einer überwiegend nicht invasiven Technologie, das heißt durch eine Magnetresonanzspektroskopie, überprüft haben. Diese Untersuchung erlaubt es, die Glukose- und Fettspeicherung sowie den Energiestoffwechsel der Mitochondrien beim Menschen zu verfolgen.

Worin lag die Aufgabe des Zentrums München bei dieser Studie? Hrabě de Angelis: Wir haben die Untersuchungen parallel im Mausmodell gemacht, da die Zugänglichkeit zu Bioproben hier besser gegeben ist. Die Mäuse erhielten ebenfalls einmal Palmöl. Danach konnten wir bei den Tieren analysieren, welche Gene in der Leber abgelesen werden. Es zeigte sich, dass die Palmölgabe die Expression von Genen verändert, die entzündliche und schützende Stoffwechselwege regulieren. Die Veränderungen des Stoffwechsels nach der Hochfettdiät in Maus und Mensch waren nahezu identisch, was die Übertragbarkeit der Ergebnisse dieses Experimentes unterstreicht.

\section{Was ist die Herausforderung bei einem solchen Projekt?}

Roden: Die Kooperation mit den Kollegen. Nur durch eine enge persönliche Abstimmung können komplexe Fragestellungen auf verschiedenen wissenschaftlichen Ebenen beantwortet werden. Mit unseren Kollegen in Portugal und besonders innerhalb des DZD vom Helmholtz Zentrum München funktioniert das aber ganz ausgezeichnet.

\section{Prof. Dr. Michael Roden}

Klinik für Endokrinologie und Diabetologie am Universitätsklinikum Düsseldorf und Deutsches Diabetes-Zentrum (DDZ) sowie Vorstand des Deutschen Zentrums für Diabetesforschung

Auf'm Hennekamp 65, 40225 Düsseldorf

E-mail-Adresse: michael.roden@DDZ.uni-duesseldorf.de

Prof. Dr. Martin Hrabě de Angelis

Vorstand des DZD und Direktor Instituts für Experimentelle Genetik

(IEG) am Helmholtz Zentrum München

Ingolstädter Landstraße 1, 85764 Neuherberg

E-mail-Adresse: hrabe@helmholtz-muenchen.de

Grundlagenforschung, Epidemiologie und Klinik -

Das Deutsche Zentrum für Diabetesforschung im Überblick

Im Deutschen Zentrum für Diabetesforschung (DZD) arbeiten Experten aus Grundlagenforschung, Epidemiologie und klinischer Anwendung deutschlandweit zusammen. Durch diesen translationalen Forschungsansatz können Beobachtungen aus epidemiologischen Studien im Labor überprüft und die Ergebnisse aus dem Labor schneller in die klinische Anwendung überführt werden. Ziel des DZD ist es, die Erkenntnisse der Diabetesforschung möglichst schnell zum Patienten zu bringen, um Diabetes vorzubeugen und zu behandeln sowie Folgeerkrankungen zu vermeiden.

Partner im DZD sind das Deutsche Diabetes-Zentrum in Düsseldorf, das Deutsche Institut für Ernährungsforschung in Potsdam-Rehbrücke, das Helmholtz Zentrum München - Deutsches Forschungszentrum für Gesundheit und Umwelt, das Institut für Diabetesforschung und Metabolische Erkrankungen des Helmholtz Zentrum München an der Eberhard-Karls-Universität Tübingen und das Paul-Langerhans-Institut Dresden des Helmholtz Zentrum München am Universitätsklinikum Carl Gustav Carus der Technischen Universität Dresden.

Zur Komplettierung und Stärkung der wissenschaftlichen Kompetenz integrierte das DZD einzelne Diabeteswissenschaftler und -forschungsgruppen an den Universitäten in Heidelberg, Köln, Leipzig, München und Lübeck als Assoziierte Partner. Weiterhin sind Wissenschaftler des ehemaligen Kompetenznetzes Diabetes mellitus Projektpartner im DZD.

Weitere Infos: www.dzd-ev.de 